TRANSACTIONS OF THE

AMERICAN MATHEMATICAL SOCIETY

Volume 339, Number 2, October 1993

\title{
WHEN CANTOR SETS INTERSECT THICKLY
}

\author{
BRIAN R. HUNT, ITTAI KAN, AND JAMES A. YORKE
}

\begin{abstract}
The thickness of a Cantor set on the real line is a measurement of its "size". Thickness conditions have been used to guarantee that the intersection of two Cantor sets is nonempty. We present sharp conditions on the thicknesses of two Cantor sets which imply that their intersection contains a Cantor set of positive thickness.
\end{abstract}

\section{INTRODUCTION}

Newhouse defined [5] a nonnegative quantity called the "thickness" of a Cantor set in order to formulate conditions which will guarantee that two Cantor sets intersect. (All Cantor sets considered in this paper lie in $\mathbb{R}^{1}$.) These conditions have been used $[5,6,7,8,9]$ in the study of two-dimensional dynamical systems to deduce the existence of tangencies between stable and unstable manifolds whose one-dimensional cross sections are Cantor sets.

Thickness may be thought of as a measure of how large a Cantor set is relative to the intervals in its complement. Henceforth, these intervals will be referred to as gaps; the two unbounded intervals in the complement are each included in our use of the term gap. Newhouse's result $[5,7,8]$ is that two Cantor sets must intersect if the product of their thicknesses is at least one, and neither set lies in a gap of the other. When this latter condition is satisfied, the sets are said to be interleaved. In [10], Williams observed the surprising fact that two interleaved Cantor sets can have thicknesses well above one and still only intersect in a single point. One might hope that under sufficiently strong thickness conditions, the intersection would be a Cantor set. However, the intersection of two arbitrarily thick interleaved Cantor sets can contain isolated points, so Williams posed the question of what conditions on the thicknesses of two interleaved Cantor sets will guarantee that their intersection contains another Cantor set. Williams obtained such a condition, though it is not sharp. In this paper we obtain the sharp condition. More precisely, we find a curve in $\left(\tau_{1}, \tau_{2}\right)$-space such that if the ordered pair $\left(\tau_{1}, \tau_{2}\right)$ of thicknesses of two interleaved Cantor sets lies above the curve, their intersection contains a Cantor set, but if the pair of thicknesses lies below the curve, there exist examples for which the intersection is a single point. Kraft [2] has independently arrived at this condition. We

Received by the editors July 9, 1990 and, in revised form, July 23, 1991.

1991 Mathematics Subject Classification. Primary 28A80, 51F99.

The first author was supported by the ONT Postdoctoral Fellowship Program administered by ASEE, by ONR, and by the NSWC Independent Research Program. All three authors were partially supported by the Applied and Computational Mathematics Program of DARPA. 


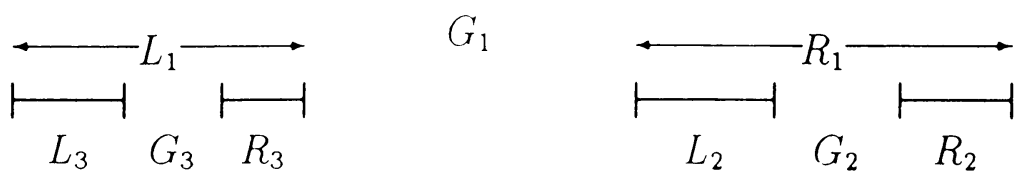

Figure 1. Constructing a Cantor set

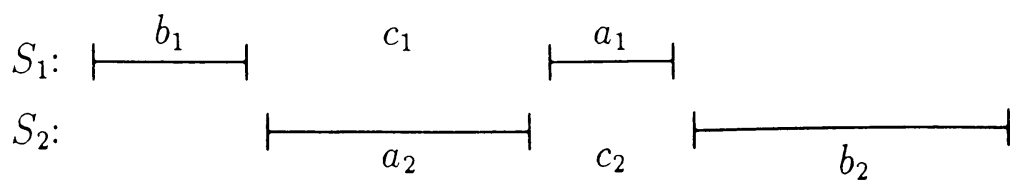

FIGURE 2. Nonintersecting interleaved sets

further show that if the thickness pair lies above the curve, the intersection must contain a Cantor set of positive thickness. This is the only result that addresses in terms of thickness how large the intersection of two Cantor sets must be. There are well-known probabilistic results concerning the Hausdorff dimensions of intersections of Cantor sets (cf. [1, 3, 4]).

One may think of a Cantor set as being constructed by starting with a closed interval and successively removing open gaps in order of decreasing length. Williams' formulation of the thickness of a Cantor set may then be thought of as follows. Each gap $G_{n}$ is removed from a closed interval $I_{n}$, leaving behind closed intervals $L_{n}$, the left piece of $I_{n}-G_{n}$, and $R_{n}$ on the right (see Figure 1). Let $\rho_{n}$ be the ratio of the length of the smaller of $L_{n}$ and $R_{n}$ to the length of $G_{n}$. The thickness of the set is the infimum of $\rho_{n}$ over all $n$.

We consider as an example the "middle-third" Cantor set, constructed as follows. Start with the closed interval $[0,1]$, and remove the open interval $(1 / 3,2 / 3)$, the middle third of the original interval. Then from each of the two remaining intervals, remove their middle thirds; repeat this process infinitely often. Each gap $G_{n}$ is the same length as the adjacent intervals $L_{n}$ and $R_{n}$, so $\rho_{n}=1$ for all $n$. Thus the thickness of the middle-third Cantor set is one.

There is a connection between the thickness of a Cantor set and its fractal dimension, which depends in part on how the ratios $\rho_{n}$ are distributed as $n \rightarrow \infty$. However, two large gaps close together make the thickness of a set very small, while its dimension can still be large. It was shown in [7] that the Hausdorff dimension of a Cantor set with thickness $\tau$ is bounded below by $\log 2 / \log (2+1 / \tau)$. This lower bound is sharp for the middle-third Cantor set (whose dimension is $\log 2 / \log 3$ ).

We offer here a new formulation of the definition of thickness which we state for all compact sets, not just Cantor sets. (The results in this and previous papers are found to be valid for all compact sets.) We define nondegenerate intervals to have infinite thickness, while singletons are defined to have thickness zero. In fact, any set containing an isolated point will be seen to have thickness zero. To define the thickness of a compact set $S$ which is not an interval, we consider a type of subset of $S$ obtained by intersecting $S$ with a closed interval. We call such an intersection $P$ a chunk of $S$ if $P$ is a proper subset of $S$ and has a positive distance from $S-P$, the complement of $P$ in $S$. (Notice that for $P$ to be a chunk both $P$ and $S-P$ must be closed and nonempty.) We then define the thickness of $S$ to be the infimum over all chunks $P$ of the 
ratio between the diameter of $P$ and the distance from $P$ to $S-P$. In the case of the middle-third Cantor set, the given ratio can be shown to be smallest when the chunk $P$ is obtained by intersecting $S$ with an interval $L_{n}$ or $R_{n}$, in which case the ratio is one. In $\S 2$ we will show that our new definition is equivalent to the old one for all Cantor sets.

The reason thickness is an appropriate quantity for determining when one can guarantee that two compact sets intersect is illustrated by considering an example where each of the two sets is an union of two disjoint intervals. For $i=1,2$ let $S_{i}$ consist of closed intervals of lengths $a_{i}$ and $b_{i}$ with $a_{i} \leq b_{i}$, separated by a distance $c_{i}$. Then each $S_{i}$ has only two chunks, and is found to have thicknesses $a_{i} / c_{i}$. If the product of the thickness $a_{1} a_{2} / c_{1} c_{2}$ is at least one, then either $a_{1} \geq c_{2}$ or $a_{2} \geq c_{1}$ (or both); assume $a_{1} \geq c_{2}$. Then since $b_{1} \geq a_{1}$, neither interval of $S_{1}$ can lie in the gap of $S_{2}$; hence if the two sets are interleaved, they must intersect. If on the other hand $a_{1} a_{2} / c_{1} c_{2}<1$, then with an affine map, we can scale the sets so that $a_{1}<c_{2}$ and $a_{2}<c_{1}$, and position them so that the component of $S_{1}$ with length $a_{1}$ lies inside the gap of $S_{2}$, and vice versa. The two sets are then interleaved, but they do not intersect (see Figure 2). This example could of course be made to involve Cantor sets by constructing very thick Cantor sets in each interval of each $S_{i}$.

An important point which is apparent in the above example is that the union of two sets can have a smaller thickness than either of the original sets. In other words, adding points to a set can decrease its thickness. By the same token, one may be able to increase the thickness of a set by removing appropriate subsets. This observation is useful in the following way. No matter how thick two interleaved compact sets are, their intersection may have thickness zero because it may contain isolated points, or arbitrarily small chunks which are relatively isolated from the rest of the intersection. Nonetheless we are able to show that if the original sets are thick enough, then by throwing out the relatively isolated parts of their intersection we can obtain a set of positive thickness in the intersection.

To define the set $C$ of thickness pairs $\left(\tau_{1}, \tau_{2}\right)$ for which a Cantor set of intersection can be guaranteed, we make use of the functions

$$
f(\tau)=\frac{\tau^{2}+3 \tau+1}{\tau^{2}}, \quad g(\tau)=\frac{(2 \tau+1)^{2}}{\tau^{3}} .
$$

Let $C$ be the set of pairs $\left(\tau_{1}, \tau_{2}\right)$ for which one of the following sets of conditions holds:

$$
\tau_{1} \geq \tau_{2}, \quad \tau_{1}>f\left(\tau_{2}\right), \quad \text { and } \quad \tau_{2}>g\left(\tau_{1}\right)
$$

or

$$
\tau_{2} \geq \tau_{1}, \quad \tau_{2}>f\left(\tau_{1}\right), \quad \text { and } \quad \tau_{1}>g\left(\tau_{2}\right)
$$

(see Figure 3). Our main result is as follows.

Theorem 1. There is a function $\varphi\left(\tau_{1}, \tau_{2}\right)$ which is positive in region $C$ such that for all interleaved compact sets $S_{1}, S_{2} \subset \mathbb{R}$ with $\tau\left(S_{1}\right) \geq \tau_{1}$ and $\tau\left(S_{2}\right) \geq \tau_{2}$, there is a set $S \subset S_{1} \cap S_{2}$ with thickness at least $\varphi\left(\tau_{1}, \tau_{2}\right)$.

Notice that a compact set with positive thickness can have no isolated points, and thus must either be a Cantor set or contain an interval; either way it contains a Cantor set. 


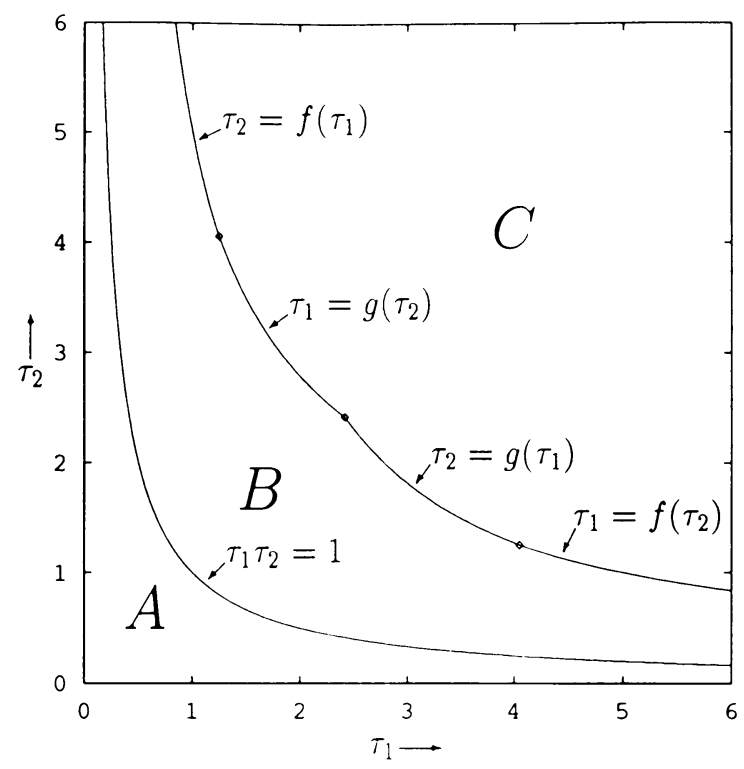

Figure 3. The intersection of two interleaved compact sets with thicknesses $\tau_{1}$ and $\tau_{2}$ can be empty for $\left(\tau_{1}, \tau_{2}\right)$ in region $A$, must be nonempty but can be a single point in region $B$, and must contain a set of positive thickness in region $C$.

We remark that $\left(\tau_{1}, \tau_{2}\right)$ is in $C$ if both thicknesses are greater than $\sqrt{2}+1$. This is the critical value Williams found for the case of interleaved Cantor sets with the same thickness. Also, no matter how small one thickness is, the other thickness can be chosen large enough so that the pair lies in $C$. Our results and the results of Newhouse are summarized in Figure 3.

In $\S 2$ we give a proof of Newhouse's result, which will illustrate some of the methods to be used later. Then we present for all pairs $\left(\tau_{1}, \tau_{2}\right)$ not in $C$ an example of interleaved compact sets with thicknesses $\tau_{1}$ and $\tau_{2}$ whose intersection is a single point (except when $\left(\tau_{1}, \tau_{2}\right)$ is on the boundary of $C$, in which case our example gives a countable intersection). This example shows that Theorem 1 is sharp in that its conclusion cannot hold for any larger set of thickness pairs $\left(\tau_{1}, \tau_{2}\right)$. In $\S 3$ we prove Theorem 1 , and in $\S 4$ we discuss some further properties of $S_{1} \cap S_{2}$. The positive thickness set $S \in S_{1} \cap S_{2}$ constructed in $\S 3$ need not be dense in $S_{1} \cap S_{2}$; however we find that there are subsets with thickness at least $\varphi\left(\tau_{1}, \tau_{2}\right)$ arbitrarily near any accumulation point of $S_{1} \cap S_{2}$. In addition, we find bounds on the diameter of $S$ which allow us to obtain thickness conditions that imply that the intersection of three Cantor sets is nonempty.

\section{Preliminaries}

Let us define precisely the concepts and notation we will use.

Definition 1. We say two sets $S_{1}, S_{2} \subset \mathbb{R}$ are interleaved if each set intersects the interior of the convex hull of the other set (that is, neither set is contained in the closure of a gap of the other set). 

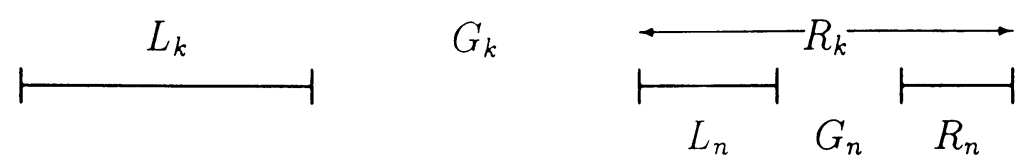

Figure 4. Chunks and gaps of a Cantor set $(k<n)$

We define the distance between two nonempty sets $S_{1}, S_{2}$ to be

$$
d\left(S_{1}, S_{2}\right)=\inf \left\{|x-y| \mid x \in S_{1}, y \in S_{2}\right\},
$$

and write $S_{2}-S_{1}$ for the intersection of $S_{2}$ with the complement of $S_{1}$. We say that a set $S_{1}$ is a chunk of a set $S_{2}$, and write $S_{1} \propto S_{2}$, if $S_{1}$ is the intersection of a closed interval with $S_{2}$, is a proper subset of $S_{2}$, and $d\left(S_{1}, S_{2}-S_{1}\right)>0$. Notice that a closed set $S$ has a chunk if and only if it is not connected. We denote the diameter of a set $S$ (the length of its convex hull) by $|S|$.

Definition 2. Given a compact set $S \subset \mathbb{R}$, we define the thickness of $S$ to be

$$
\tau(S)=\inf _{P \propto S} \frac{|P|}{d(P, S-P)}
$$

provided $S$ has a chunk. Otherwise, we let $\tau(S)=0$ if $S$ is empty or consists of a single point, and $\tau(S)=\infty$ if $S$ is an interval with positive length.

The following simple proposition demonstrates that Definition 2 agrees with Williams' definition of thickness for Cantor sets [10].

Proposition 2. Let $S$ be a Cantor set, and define the ratios $\rho_{n}$ as in the introduction. Then the quantity $\tau(S)$ given by (2.1) is equal to the infimum of $\rho_{n}$ over all $n$.

Proof. The intervals $L_{n}$ and $R_{n}$ defined in the introduction are the convex hulls of chunks $A_{n}=L_{n} \cap S$ and $B_{n}=R_{n} \cap S$ of $S$. Since the gap $G_{n}$ is not larger than any previously removed gap $G_{k}, k<n$, it follows that

$$
d\left(A_{n}, S-A_{n}\right)=d\left(B_{n}, S-B_{n}\right)=\left|G_{n}\right|
$$

(see Figure 4). Thus for all $n$,

$$
\rho_{n}=\min \left(\frac{\left|L_{n}\right|}{\left|G_{n}\right|}, \frac{\left|R_{n}\right|}{\left|G_{n}\right|}\right)=\min \left(\frac{\left|A_{n}\right|}{d\left(A_{n}, S-A_{n}\right)}, \frac{\left|B_{n}\right|}{d\left(B_{n}, S-B_{n}\right)}\right) \geq \tau(S) .
$$

Next, if $P$ is a chunk of $S$, it must be bordered on each side by a gap of $S$; let $G_{n}$ be the smaller of these two gaps. Then $\left|G_{n}\right|=d(P, S-P)$ and $|P| \geq \min \left(\left|L_{n}\right|,\left|R_{n}\right|\right)$. Therefore

$$
\tau(S)=\inf _{P \propto S} \frac{|P|}{d(P, S-P)} \geq \inf _{n} \rho_{n},
$$

which completes the proof.

We now prove Newhouse's result in a way that will motivate our later examples and methods. 


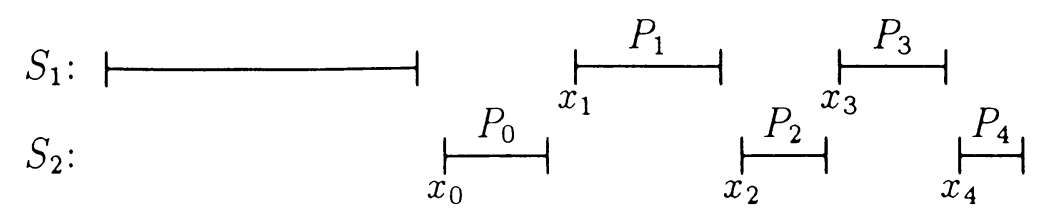

FIGURE 5. The points $x_{n}$ and chunks $P_{n}$

Proposition 3. If $S_{1}$ and $S_{2}$ are interleaved compact sets with $\tau\left(S_{1}\right) \cdot \tau\left(S_{2}\right) \geq 1$, then $S_{1} \cap S_{2}$ is not empty.

Proof. Let $S_{1}$ and $S_{2}$ be as above, and let

$$
x_{0}=\max \left(\inf _{x \in S_{1}} x, \inf _{x \in S_{2}} x\right),
$$

the greater of the leftmost points of $S_{1}$ and $S_{2}$. Assume without loss of generality that $x_{0} \in S_{2}$. We will show that $S_{1} \cap S_{2}$ is nonempty by looking for the leftmost point of this set. Let $x_{1}$ be the leftmost point of $S_{1}$ which is at least as great as $x_{0}$. Since $S_{1}$ and $S_{2}$ are interleaved, $x_{1}$ must exist (otherwise $S_{1}$ would lie entirely to the left of $S_{2}$; see Figure 5). Next, let $x_{2}$ be the leftmost point of $S_{2}$ greater than or equal to $x_{1}$. Once again the interleaving assumption implies that $x_{2}$ exists, for otherwise $S_{2}$ would lie inside a gap of $S_{1}$. We similarly define $x_{3}, x_{4}, \ldots$; if each of these points can be shown to exist, we claim to be done. Then $\left\{x_{n}\right\}$ will be a nondecreasing sequence which is bounded above (since $S_{1}$ and $S_{2}$ are bounded), so it approaches a limit. This limit must belong to both $S_{1}$ and $S_{2}$ since these sets are closed and the odd numbered terms of $\left\{x_{n}\right\}$ belong to $S_{1}$, the even ones to $S_{2}$.

If at any step $x_{n}$ exists and equals $x_{n-1}$, then $x_{n+1}, x_{n+2}, \ldots$ will also equal $x_{n-1}$, and we will have found a point in $S_{1} \cap S_{2}$. Henceforth we assume $x_{0}<x_{1}<\cdots$ as long as they are defined. We know at least that $x_{0}, x_{1}$, and $x_{2}$ exist, so there is a chunk $P_{0}$ of $S_{2}$ which lies in $\left[x_{0}, x_{1}\right)$, whose diameter is thus less than $x_{1}-x_{0}$, and whose distance from the rest of $S_{2}$ is greater than $x_{2}-x_{1}$ (see again Figure 5). Then

$$
\frac{x_{1}-x_{0}}{x_{2}-x_{1}}>\frac{\left|P_{0}\right|}{d\left(P_{0}, S_{2}-P_{0}\right)} \geq \tau\left(S_{2}\right) \text {. }
$$

Let $P_{1}$ be the largest chunk of $S_{1}$ which lies in $\left[x_{1}, x_{2}\right)$. If $x_{3}$ did not exist, in other words if all points in $S_{1}$ were less than $x_{2}$, then $S_{1}-P_{1}$ would lie to the left of $P_{1}$, and the distance between these sets would be greater than $x_{1}-x_{0}$. But then using (2.2) and $\tau\left(S_{1}\right) \cdot \tau\left(S_{2}\right) \geq 1$ we would have

$$
\frac{\left|P_{1}\right|}{d\left(P_{1}, S_{1}-P_{1}\right)}<\frac{x_{2}-x_{1}}{x_{1}-x_{0}}<\frac{1}{\tau\left(S_{2}\right)} \leq \tau\left(S_{1}\right),
$$

contradicting the definition of the thickness of $S_{1}$. Thus $x_{3}$ exists, and similarly to (2.2) we obtain

$$
\frac{x_{2}-x_{1}}{x_{3}-x_{2}}>\frac{\left|P_{1}\right|}{d\left(P_{1}, S_{1}-P_{1}\right)} \geq \tau\left(S_{1}\right) .
$$

Likewise (2.3) can be used to show the existence of $x_{4}$, and so forth. The proof is completed by induction. 
One could similarly find the rightmost point in $S_{1} \cap S_{2}$, but as Williams observed it may coincide with the leftmost point, even if both thicknesses are significantly greater than 1 . We next present an example which will give a single point of intersection for thickness pairs $\left(\tau_{1}, \tau_{2}\right)$ not in the closure of region $C$, and a countable intersection for $\left(\tau_{1}, \tau_{2}\right)$ on the boundary of $C$. In our example both sets are countable unions of closed intervals, but they could be replaced by Cantor sets with the same thicknesses by constructing a very thick Cantor set in each of the closed intervals.

Let $\tau$ be a positive constant, and define the intervals

$$
\begin{gathered}
A_{0}=\left[\tau^{2}+3 \tau+1,(2 \tau+1)^{2}\right], \quad B_{0}=\left[\tau^{2}, \tau^{2}+3 \tau+1\right], \\
A_{n}=\left(-\frac{\tau}{2 \tau+1}\right)^{n} A_{0}, \quad B_{n}=\left(-\frac{\tau}{2 \tau+1}\right)^{n} B_{0},
\end{gathered}
$$

where multiplication of a set by a scalar means the set obtained by multiplying each element of the original set by the given scalar. Let

$$
S_{1}=\left(\bigcup_{n=0}^{\infty} A_{n}\right) \cup\{0\}, \quad S_{2}=\left(\bigcup_{n=0}^{\infty} B_{n}\right) \cup\{0\} .
$$

Notice that $B_{n}$ is the closure of the interval between $A_{n}$ and $A_{n+2}$ for all $n$, and $A_{n}$ is the closure of the interval between $B_{n-2}$ and $B_{n}$ for $n \geq 2$. Thus $S_{1} \cap S_{2}$ is countable, containing only the point 0 and endpoints of the intervals $A_{n}$ and $B_{n}$. Furthermore, the intersection could by reduced to only the point 0 by shrinking the intervals which make up one of the sets by a factor arbitrarily close to one.

Let us compute the thicknesses of the sets $S_{1}$ and $S_{2}$. Observe that

$$
\begin{gathered}
\left|A_{n}\right|=d\left(B_{n-2}, B_{n}\right)=\left(\frac{\tau}{2 \tau+1}\right)^{n} \tau(3 \tau+1), \\
\left|B_{n}\right|=d\left(A_{n}, A_{n+2}\right)=\left(\frac{\tau}{2 \tau+1}\right)^{n}(3 \tau+1) .
\end{gathered}
$$

The intervals $A_{n}$ are ordered from left to right $A_{1}, A_{3}, A_{5}, \ldots, A_{4}, A_{2}, A_{0}$, so any chunk $P$ of $S_{1}$ which does not contain 0 must be a finite union of consecutive even or odd numbered $A_{n}$. Let $A_{n}$ be the interval in $P$ with the largest index; then

$$
\frac{|P|}{d\left(P, S_{1}-P\right)} \geq \frac{\left|A_{n}\right|}{d\left(A_{n}, A_{n+2}\right)}=\tau,
$$

with equality holding when $P=A_{n}$. On the other hand, if a chunk $P$ of $S_{1}$ contains zero, let $n$ be the larger index of the leftmost and rightmost $A_{k}$ in $P$. Then $P$ must contain $A_{n-1}$, and since $P$ is not all of $S_{1}, n \geq 2$, so

$$
\frac{|P|}{d\left(P, S_{1}-P\right)} \geq \frac{\left|A_{n} \cup A_{n-1}\right|}{d\left(A_{n}, A_{n-2}\right)}=\frac{(\tau /(2 \tau+1))^{n-1}(3 \tau+1)(2 \tau+1)}{(\tau /(2 \tau+1))^{n-2}(3 \tau+1)}=\tau \text {. }
$$

Therefore the thickness of $S_{1}$ is $\tau$.

Similarly, if $P$ is a chunk of $S_{2}$, then for an appropriately chosen $B_{n}$, either

$$
\frac{|P|}{d\left(P, S_{2}-P\right)} \geq \frac{\left|B_{n}\right|}{d\left(B_{n}, B_{n+2}\right)}=\frac{(2 \tau+1)^{2}}{\tau^{3}}=g(\tau)
$$


or

$$
\begin{aligned}
\frac{|P|}{d\left(P, S_{2}-P\right)} & \geq \frac{\left|B_{n} \cup B_{n-1}\right|}{d\left(B_{n}, B_{n-2}\right)} \\
& =\frac{(\tau /(2 \tau+1))^{n-1}((3 \tau+1) /(2 \tau+1))\left(\tau^{2}+3 \tau+1\right)}{(\tau /(2 \tau+1))^{n-2} \tau(3 \tau+1)} \\
& =\frac{\tau^{2}+3 \tau+1}{\tau^{2}}=f(\tau) .
\end{aligned}
$$

Thus

$$
\tau\left(S_{2}\right)=\min (f(\tau), g(\tau))
$$

As we pointed out before, by reducing the thickness of $S_{2}$ by an arbitrarily small amount we can shrink the intersection of $S_{1}$ and $S_{2}$ to a single point. Let $\tau_{1}$ denote the thickness of the set $S_{1}$, and let $\tau_{2}$ be the thickness of $S_{2}$. Then up to a change of indices, the above construction demonstrates that a single point of intersection can be obtained when either

$$
\tau_{1}<\min \left(f\left(\tau_{2}\right), g\left(\tau_{2}\right)\right)
$$

or

$$
\tau_{2}<\min \left(f\left(\tau_{1}\right), g\left(\tau_{1}\right)\right) .
$$

Also, if either (2.4) or (2.5) is an equality instead, the intersection can be countable. (Kraft [2] has analyzed this borderline case and determined when the intersection can be finite.) Therefore we can only hope to guarantee an uncountable intersection if

$$
\tau_{1}>\min \left(f\left(\tau_{2}\right), g\left(\tau_{2}\right)\right)
$$

and

$$
\tau_{2}>\min \left(f\left(\tau_{1}\right), g\left(\tau_{1}\right)\right) .
$$

One may check that $g(\tau)>f(\tau)>\sqrt{2}+1$ for $\tau<\sqrt{2}+1$ and $g(\tau)<f(\tau)<$ $\sqrt{2}+1$ for $\tau>\sqrt{2}+1$. Therefore (2.6) and (2.7) are equivalent to (1.1) in the case $\tau_{1} \geq \tau_{2}$, and to (1.2) when $\tau_{2} \geq \tau_{1}$.

\section{PROOF OF MAIN RESUlT}

We now prove Theorem 1 by constructing a set $S$ with positive thickness in $S_{1} \cap S_{2}$.

Proof of Theorem 1. Let $S_{1}$ and $S_{2}$ be interleaved compact sets with $\tau\left(S_{1}\right) \geq \tau_{1}$ and $\tau\left(S_{2}\right) \geq \tau_{2}$ for some $\left(\tau_{1}, \tau_{2}\right)$ in region $C$ of Figure 3 . Let the gaps of $S_{1}$ be $I_{0}, I_{1}, I_{2}, \ldots$, with $I_{0}$ and $I_{1}$ unbounded, $I_{0}$ to the left of $I_{1}$, and $\left|I_{2}\right| \geq\left|I_{3}\right| \geq \cdots$. For $S_{2}$ we define $J_{0}, J_{1}, J_{2}, \ldots$ similarly. We refer to the intervals $I_{n}$ and $J_{n}$ collectively as the "original gaps". Our goal is to construct the complement of $S$ as a union of disjoint open intervals $K_{0}, K_{1}, K_{2}, \ldots$ with $K_{0}$ and $K_{1}$ unbounded, and with every original gap contained in some $K_{m}$ (whence $S \subset S_{1} \cap S_{2}$ ). To get a lower bound on the thickness of $S$, observe that every chunk $P$ of $S$ is bordered on each side by a gap of $S$, with at least one of the bordering gaps being bounded. Pick a chunk $P$, and say $P$ is bordered by $K_{m}$ and $K_{n}$ with $m>n$ and $m \geq 2$. Then

$$
\frac{|P|}{d(P, S-P)}=\frac{d\left(K_{m}, K_{n}\right)}{\min \left(\left|K_{m}\right|,\left|K_{n}\right|\right)} \geq \frac{d\left(\dot{K}_{m}, K_{n}\right)}{\left|K_{m}\right|} \text {. }
$$




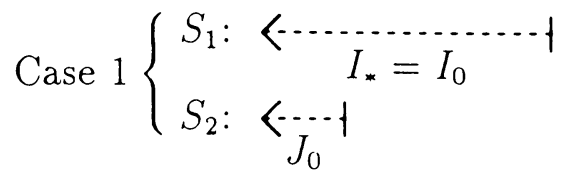

$$
\begin{aligned}
& \begin{array}{l}
|\cdots\rangle\rangle \\
I_{1} \\
J_{*}=J_{1}
\end{array}
\end{aligned}
$$

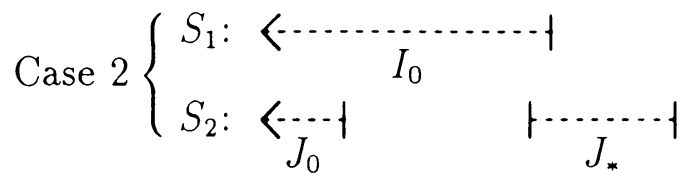

$$
\begin{aligned}
& \begin{array}{r}
|\cdots\rangle \\
\left.I_{*}=I_{1}-\cdots\right\rangle \\
J_{1}
\end{array}
\end{aligned}
$$

FIGURE 6. Cases in the construction of $I_{*}$ and $J_{*}$

The theorem will therefore be proved when we show for some $\varphi\left(\tau_{1}, \tau_{2}\right)>0$ that whenever $m>n$ and $m \geq 2$,

$$
\frac{d\left(K_{m}, K_{n}\right)}{\left|K_{m}\right|} \geq \varphi\left(\tau_{1}, \tau_{2}\right) .
$$

We begin by finding a pair of original gaps $I_{*}$ and $J_{*}$ between which $S$ will lie; that is, $I_{*}$ and $J_{*}$ will be contained in $K_{0}$ and $K_{1}$. The properties we desire of $I_{*}$ and $J_{*}$ are that they are a positive distance apart, that all gaps of $S_{1}$ with an endpoint between the closures of $I_{*}$ and $J_{*}$ are bounded and no larger than $I_{*}$, and likewise (in comparison to $J_{*}$ ) for gaps of $S_{2}$ between $I_{*}$ and $J_{*}$. We will show later that once $I_{*}$ and $J_{*}$ have been determined, the diameter of $S$ can be bounded below by a constant depending on $\tau_{1}$ and $\tau_{2}$ times the distance between $I_{*}$ and $J_{*}$.

Assume without loss of generality that $J_{0} \subset I_{0}$. If $I_{1} \subset J_{1}$ (Case 1 of Figure 6), then $I_{*}=I_{0}$ and $J_{*}=J_{1}$ have the above properties; they must be separated by a positive distance since $S_{1}$ and $S_{2}$ are interleaved. If $J_{1} \subset I_{1}$ (Case 2 of Figure 6), let $J_{*}$ be the largest gap of $S_{2}$ with an endpoint between $I_{0}$ and $I_{1}$, and let $I_{*}$ be whichever of $I_{0}$ and $I_{1}$ is farthest from $J_{*}$. At least one of $I_{0}$ and $I_{1}$ must be a positive distance from $J_{*}$ since $S_{1}$ and $S_{2}$ are interleaved.

Next, let $t$ be a positive constant whose precise value will be chosen later; for now we assume that $t<\left(\tau_{1} \tau_{2}-1\right) /\left(\tau_{1}+\tau_{2}+2\right)<\min \left(\tau_{1}, \tau_{2}\right)$. Assume without loss of generality that $I_{*}$ lies to the left of $J_{*}$. We begin constructing $K_{0}$ by requiring that it contain $I_{*}$. We then require that $K_{0}$ contain the rightmost bounded $J_{n}$ with $d\left(I_{*}, J_{n}\right) \leq t\left|J_{n}\right|$ (we will verify that there is a rightmost gap satisfying this condition when we later examine our construction in more detail). If there does not exist such a $J_{n}$ that is not already contained in $I_{*}$, we stop the construction and let $K_{0}=I_{*}$. Otherwise, we further require that $K_{0}$ contain the rightmost bounded $I_{m}$ that is within $t$ times its length of the previously added $J_{n}$. Again, if this requirement does not extend $K_{0}$ any farther rightward, we stop the construction. If not, we then add to $K_{0}$ the rightmost $J_{l}$ which is within $t$ times its length of $I_{m}$ and is at most as large as $J_{n}$ (see Figure 7). If a next step is necessary, we consider gaps of $S_{1}$ which are no larger than $I_{m}$, and so forth. We may have to continue this process infinitely often, but if so we must converge to a right endpoint for $K_{0}$, since there is no way this construction can extend past the rightmost point in $S_{1} \cup S_{2}$.

We define $K_{1}$ similarly, starting with the requirement that $K_{1}$ contain $J_{*}$ 


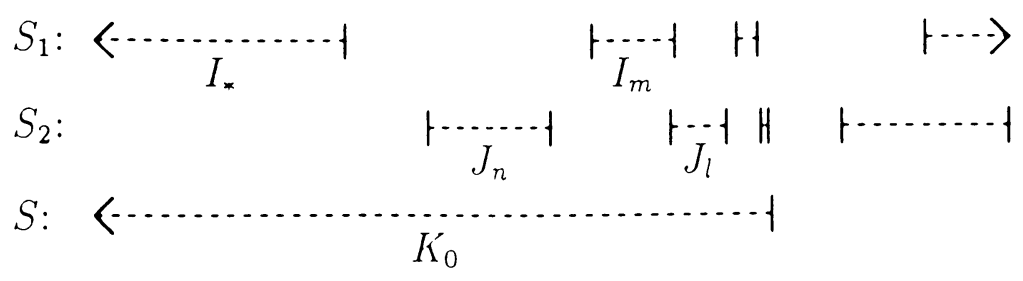

FIGURE 7. The construction of $K_{0}$

and extending $K_{1}$ to the left if necessary in the same way we constructed $K_{0}$. Next, to construct $K_{2}$ we first require that it contain the largest original gap (choose any one in the case of a tie) not contained in $K_{0} \cup K_{1}$ (if no such gap exists, we leave $K_{2}$ undefined and let $S$ be the complement of $\left.K_{0} \cup K_{1}\right)$. Then we extend it on both the left and right in the same manner as before, but considering only gaps that are at most as large as the one we started with, to obtain the endpoints of $K_{2}$. We next start with the largest original gap not contained in $K_{0} \cup K_{1} \cup K_{2}$, proceeding similarly to define $K_{3}$, and so forth. Any given original gap must eventually be contained in some $K_{n}$ because there can be only finitely many original gaps that are as large or larger than the given one. We do not yet know that the $K_{n}$ are disjoint from each other; this will follow when we prove (3.1), though.

Let us now examine our construction more closely. Define $l(I)$ and $r(I)$ to be respectively the left and right endpoints of an interval $I$. For a given $K_{n}$, let $G_{0}$ be the gap we started with in its construction, which for $n \geq 2$ must be the largest original gap it contains (or at least tied for the largest). For simplicity we assume here that $G_{0}$ is a gap of $S_{1}$. Consider the collection $E$ of all $J_{n}$ with $\left|J_{n}\right| \leq\left|G_{0}\right|, r\left(J_{n}\right)>r\left(G_{0}\right)$, and $d\left(G_{0}, J_{n}\right) \leq t\left|J_{n}\right|$. We claim that the members of $E$ (if any) are increasing in size from left to right. If $J_{m}, J_{n} \in E$ with $J_{m}$ to the left of $J_{n}$, then since $r\left(J_{m}\right)<r\left(J_{n}\right)$, it follows that $d\left(J_{m}, J_{n}\right)<$ $d\left(G_{0}, J_{n}\right) \leq t\left|J_{n}\right|$. Since $t<\tau_{2}$ and $d\left(J_{m}, J_{n}\right) \geq \tau_{2} \min \left(\left|J_{m}\right|,\left|J_{n}\right|\right)$, it must then be the case that $\left|J_{n}\right|>\left|J_{m}\right|$. Thus if $E$ is not empty, it must have a rightmost member, which we call $G_{1}$ (notice that $G_{1}$ is also the largest member of $E$ ). If $E$ is empty, we let $G_{1}$ be empty, but in order to facilitate future formalism, we define $\left|G_{1}\right|=0$ and $r\left(G_{1}\right)=r\left(G_{0}\right)$. One must keep in mind this degenerate case in verifying the assertions and formulas that follow.

We likewise define $G_{2}$ to be the rightmost gap of $S_{1}$ which is at most as large as $G_{0}$ and lies within $t$ times its length of $G_{1}$; again if no such gap exists with $r\left(G_{2}\right)>r\left(G_{1}\right)$ we say that $\left|G_{2}\right|=0$ and $r\left(G_{2}\right)=r\left(G_{1}\right)$. Next, to define $G_{3}$ we consider only gaps of $S_{2}$ which are at most as large as $G_{1}$, for $G_{4}$ we look only at gaps of $S_{1}$ no larger than $G_{2}$, and so forth. Define $G_{-1}, G_{-2}, \ldots$ similarly to be the leftmost (and largest) gaps added to $K_{n}$ at each stage of the process of extending $K_{n}$ leftward. Then we may think of the open interval $K_{n}$ as being defined by

$$
l\left(K_{n}\right)=\lim _{m \rightarrow-\infty} l\left(G_{m}\right), \quad r\left(K_{n}\right)=\lim _{m \rightarrow \infty} r\left(G_{m}\right) .
$$

Each limit exists because it is the limit of a bounded monotonic sequence.

In the above construction, the even-numbered $G_{m}$ are gaps of $S_{1}$ and the odd-numbered ones are gaps of $S_{2}$, but if $G_{0}$ had been a gap of $S_{2}$ it would be the other way around. In any case, $G_{0}$ is the largest even-numbered $G_{m}$ and either $G_{1}$ or $G_{-1}$ is the largest odd-numbered one. Also, the even-numbered 
$G_{m}$ decrease monotonically in size as one moves either rightward or leftward from the largest, and the same statement holds for the odd-numbered $G_{m}$. We call a given $G_{m}$ either a "1-gap" or "2-gap" of $K_{n}$ according to whether it is a gap of $S_{1}$ or $S_{2}$. Notice that not all original gaps contained in $K_{n}$ are 1-gaps or 2-gaps, only those that have been given a label $G_{m}$ in the construction of $K_{n}$. When we refer henceforth to left-to-right ordering or adjacency among the 1-gaps and 2-gaps of a given $K_{n}$, it is with respect to the ordering $\ldots, G_{-2}, G_{-1}, G_{0}, G_{1}, G_{2}, \ldots$ (Thus, for instance, 1-gaps can only be adjacent to 2-gaps and vice versa.)

The following lemma will be used in bounding both the numerator and denominator of the left side of (3.1). It establishes for all $m \geq 0$ a bound on how far $K_{n}$ can extend to the right of $G_{m}$ in terms of how far $G_{m+1}$ extends past $G_{m}$, and similarly for $m \leq 0$ on the left.

Lemma 4. Assume $t<\left(\tau_{1} \tau_{2}-1\right) /\left(\tau_{1}+\tau_{2}+2\right)$. Let

and

$$
\sigma_{1}=\frac{\left(\tau_{1}-t\right)\left(\tau_{2}+1\right)}{\left(\tau_{1}-t\right)\left(\tau_{2}-t\right)-(1+t)^{2}}
$$

$$
\sigma_{2}=\frac{\left(\tau_{2}-t\right)\left(\tau_{1}+1\right)}{\left(\tau_{1}-t\right)\left(\tau_{2}-t\right)-(1+t)^{2}} .
$$

Let $G$ be a 1-gap of $K_{n}$ which is at least as large as all 1-gaps of $K_{n}$ to its right. Let $H$ be the next 2-gap of $K_{n}$ to the right of $G$. Then

$$
r\left(K_{n}\right)-r(G) \leq \sigma_{2}(r(H)-r(G)) .
$$

The same statement with " 1 " and " 2 " interchanged holds, as do the corresponding results for left endpoints.

Proof. Let $I$ be the next 1-gap of $K_{n}$ to the right of $H$. Then since $|I| \leq|G|$,

$$
\tau_{1}|I| \leq d(G, I) \leq d(H, I)+r(H)-r(G) \leq t|I|+r(H)-r(G),
$$

which, because $t<\tau_{1}$, implies that

Hence

$$
|I| \leq \frac{r(H)-r(G)}{\tau_{1}-t} .
$$

$$
r(I)-r(H) \leq|I|+d(H, I) \leq(1+t)|I| \leq \frac{1+t}{\tau_{1}-t}(r(H)-r(G)) .
$$

Likewise the next rightward 2-gap of $K_{n}$ extends at most

beyond $I$, and by induction

$$
\frac{1+t}{\tau_{2}-t}(r(I)-r(H))
$$

$$
\begin{aligned}
r\left(K_{n}\right)-r(G) & =r(H)-r(G)+r(I)-r(H)+\cdots \\
& \leq\left(1+\frac{1+t}{\tau_{1}-t}+\frac{1+t}{\tau_{1}-t} \frac{1+t}{\tau_{2}-t}+\cdots\right)(r(H)-r(G)) \\
& =\sigma_{2}(r(H)-r(G)) .
\end{aligned}
$$

The geometric series converges, and the denominator of $\sigma_{2}$ is positive, because of our assumption that $t<\left(\tau_{1} \tau_{2}-1\right) /\left(\tau_{1}+\tau_{2}+2\right)$.

The next lemma builds on Lemma 4 to obtain a positive lower bound on the distance between a given $K_{m}$ and $K_{n}$, provided we can find a 1-gap of $K_{m}$ and a 2-gap of $K_{n}$ which are respectively larger than all 1-gaps and 2-gaps between them. The proof is difficult and will be handled later. 


$$
\begin{aligned}
& \longleftrightarrow K_{m} \longrightarrow \\
& \longrightarrow K_{n} \longrightarrow \\
& \text { Case } 1\left\{\begin{array}{l}
S_{1}: H \quad+\cdots \cdots+1 \\
S_{2}: H--1 \quad H=I
\end{array}\right. \\
& \text { 1......-1 } \\
& H \quad+\cdots \cdots
\end{aligned}
$$

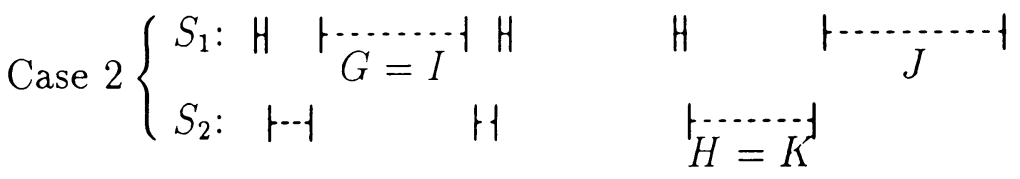

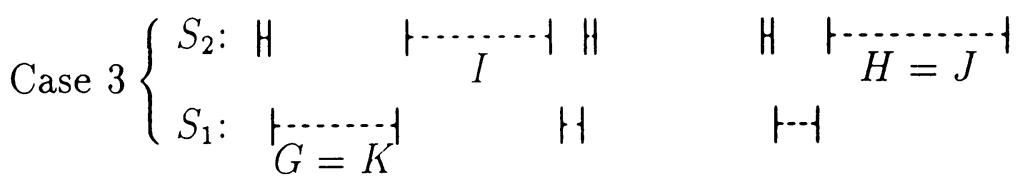

FIGURE 8. Cases in the proof of (3.1)

Lemma 5. There exists a function $\psi_{t}\left(\tau_{1}, \tau_{2}\right)$ that is positive whenever $\left(\tau_{1}, \tau_{2}\right)$ is in region $C$ and $t$ is sufficiently small, and for which the following statement holds. For $m \neq n$, let $G$ be a 1-gap of $K_{m}$ and $H$ be a 2-gap of $K_{n}$. If all 1-gaps of $K_{m}$ or $K_{n}$ with at least one endpoint between the closures of $G$ and $H$ are bounded and at most as large as $G$, and all similarly situated 2-gaps are bounded and at most as large as $H$, then

$$
d\left(K_{n}, K_{m}\right) \geq \psi_{t}\left(\tau_{1}, \tau_{2}\right) d(G, H) .
$$

Recall that to construct $K_{0}$ and $K_{1}$, we chose $I_{*}$ and $J_{*}$ to satisfy the above hypotheses. Thus we now know that $K_{0}$ and $K_{1}$ are disjoint and separated by a positive distance (which is at least $\psi_{t}\left(\tau_{1}, \tau_{2}\right)$ times the distance between $I_{*}$ and $J_{*}$ ).

Now suppose $0 \leq n<m$ and $m \geq 2$; we will prove (3.1) by finding a $G$ and $H$ which satisfy the hypotheses of Lemma 5 . Assume without loss of generality that $K_{m}$ lies to the left of $K_{n}$. Let $I$ be the largest original gap in $K_{m}$; say $I$ is a 1-gap. If all 1-gaps of $K_{n}$ are smaller than $I$ (Case 1 of Figure 8), let $H$ be the largest original gap in $K_{n}$. Since $m>n, K_{n}$ was constructed before $K_{n}$, so $H$ must be at least as large as $I$, and thus is a 2-gap. Let $G=I$; then $G$ and $H$ satisfy the hypotheses of Lemma 5. Also, $d(G, H)>t|G|$, since otherwise $G$ would have been included in the construction of $K_{n}$. If on the other hand there are 1-gaps of $K_{n}$ which are at least as large as $I$ (Cases 2 and 3 of Figure 8), let $J$ be the closest such gap to $I$. Consider all 2-gaps of $K_{m}$ or $K_{n}$ to the left of $J$; let $K$ be the largest such 2-gap (any one will do in case of a tie). Notice that $K$ must be adjacent to $I$ or $J$. If $K$ is in $K_{n}$ (Case 2), let $G=I$ and $H=K$; then $G$ and $H$ satisfy the hypotheses of Lemma 5 , and $d(G, H)>t|G|$ because $G$ was not included in $K_{n}$. Otherwise (Case 3), let $G=K$ and reverse the indices "1" and "2" (so that $G$ is a 1-gap). If the 1-gap in $K_{n}$ next to $J$ on its right is at least as large as $G$, then let $H=J$; otherwise let $H$ be the 2-gap in $K_{n}$ on the left of the leftmost 1-gap in $K_{n}$ 
which is at least as large as $G$, or let $H$ be the largest 2-gap in $K_{n}$ if there is no 1-gap in $K_{n}$ as large as $G$. Then once again, $G$ and $H$ satisfy the hypotheses of Lemma 5 and $d(G, H)>t|G|$. Notice also that in all cases, $G$ is the largest 1-gap of $K_{m}$, and $H$ is at least as large as all 2-gaps of $K_{m}$.

We now estimate how large $K_{m}$ can be. Let $I$ and $J$ be the 2-gaps of $K_{m}$ adjacent to $G$ on its left and right, respectively. Since $I$ is at most as large as $H$,

$$
\tau_{2}|I| \leq d(I, H) \leq d(I, G)+|G|+d(G, H) \leq t|I|+|G|+d(G, H),
$$

or in other words

$$
|I| \leq \frac{1}{\tau_{2}-t}(|G|+d(G, H)) .
$$

The same bound holds also for $J$, so by Lemma 4,

$$
\begin{aligned}
\left|K_{m}\right| & =|G|+l(G)-l\left(K_{m}\right)+r\left(K_{m}\right)-r(G) \\
& \leq|G|+\sigma_{2}(l(G)-l(I))+\sigma_{2}(r(J)-r(G)) \\
& \leq|G|+\sigma_{2}(1+t)(|I|+|J|) \\
& \leq|G|+2 \sigma_{2} \frac{1+t}{\left(\tau_{2}-t\right)}(|G|+d(G, H)) \\
& \leq\left(\frac{1}{t}+2 \sigma_{2} \frac{1+t}{\left(\tau_{2}-t\right)}\left(\frac{1}{t}+1\right)\right) d(G, H) \\
& =\frac{\left(\tau_{1}-t\right)\left(\tau_{2}-t\right)+(1+t)^{2}\left(2 \tau_{1}+1\right)}{t\left(\left(\tau_{1}-t\right)\left(\tau_{2}-t\right)-(1+t)^{2}\right)} d(G, H) .
\end{aligned}
$$

If on the other hand $G$ is a 2-gap and $H$ is a 1-gap, we obtain the same bound as (3.4), but with the indices " 1 " and " 2 " interchanged. Then in either case,

$$
\left|K_{m}\right| \leq \frac{\left(\tau_{1}-t\right)\left(\tau_{2}-t\right)+(1+t)^{2}\left(2 \max \left(\tau_{1}, \tau_{2}\right)+1\right)}{t\left(\left(\tau_{1}-t\right)\left(\tau_{2}-t\right)-(1+t)^{2}\right)} d(G, H) .
$$

Finally, by Lemma 5,

$$
\frac{d\left(K_{m}, K_{n}\right)}{\left|K_{m}\right|} \geq \frac{t\left(\left(\tau_{1}-t\right)\left(\tau_{2}-t\right)-(1+t)^{2}\right) \psi_{t}\left(\tau_{1}, \tau_{2}\right)}{\left(\tau_{1}-t\right)\left(\tau_{2}-t\right)+(1+t)^{2}\left(2 \max \left(\tau_{1}, \tau_{2}\right)+1\right)} .
$$

The right side of (3.5) is positive as long as $\psi_{t}\left(\tau_{1}, \tau_{2}\right)>0$ and $t$ is between 0 and $\left(\tau_{1} \tau_{2}-1\right) /\left(\tau_{1}+\tau_{2}+2\right)$, and it goes to zero when $t$ approaches any of the borderline values. Therefore the right side of (3.5) attains a maximum value, call it $\varphi\left(\tau_{1}, \tau_{2}\right)$, at some allowable value of $t$, say $t_{*}$. We thus carry out the construction of $S$ with $t=t_{*}$; then (3.1) holds, and the proof is complete.

Let $\psi\left(\tau_{1}, \tau_{2}\right)=\psi_{t_{*}}\left(\tau_{1}, \tau_{2}\right)$; then

$$
\varphi\left(\tau_{1}, \tau_{2}\right)=\frac{t_{*}\left(\left(\tau_{1}-t_{*}\right)\left(\tau_{2}-t_{*}\right)-\left(1+t_{*}\right)^{2}\right) \psi\left(\tau_{1}, \tau_{2}\right)}{\left(\tau_{1}-t_{*}\right)\left(\tau_{2}-t_{*}\right)+\left(1+t_{*}\right)^{2}\left(2 \max \left(\tau_{1}, \tau_{2}\right)+1\right)} .
$$

Remark. We will see in the proof of Lemma 5 that $\psi\left(\tau_{1}, \tau_{2}\right)$, and hence $\varphi\left(\tau_{1}, \tau_{2}\right)$, must be very small when $\left(\tau_{1}, \tau_{2}\right)$ is near the boundary of region $C$. However, if both $\tau_{1}$ and $\tau_{2}$ are large and $t$ is small compared with the two thicknesses, it is not hard to check that $\psi_{t}\left(\tau_{1}, \tau_{2}\right)$ is close to one. Then 


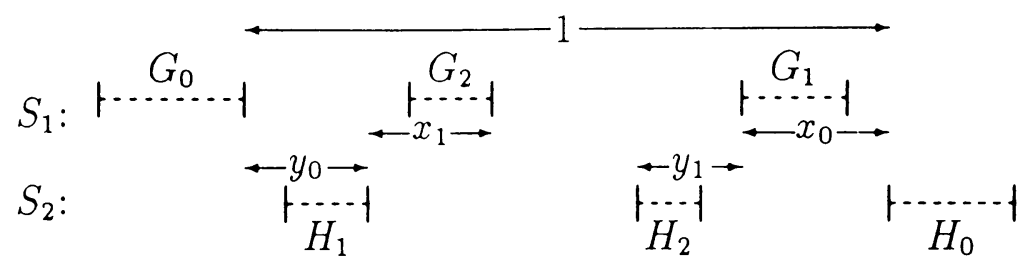

Figure 9. The gaps $G_{i}$ and $H_{i}$

if $\tau_{1}, \tau_{2} \gg 1$, one finds that $t_{*}$ is of order $\sqrt{\min \left(\tau_{1}, \tau_{2}\right)}$, whence $\varphi\left(\tau_{1}, \tau_{2}\right)$ is of order $\sqrt{\min \left(\tau_{1}, \tau_{2}\right)}$ also. Thus when the thicknesses of $S_{1}$ and $S_{2}$ are large, the lower bound we obtain on the thickness of $S$ is reasonably large.

We now prove our main technical lemma.

Proof of Lemma 5. Let $G$ be a 1-gap of $K_{m}$ and $H$ be a 2-gap of $K_{n}$ satisfying the hypotheses. We assume without loss of generality that $\tau_{1} \geq \tau_{2}$; then by (1.1) the condition $\left(\tau_{1}, \tau_{2}\right) \in C$ implies

$$
\tau_{1}>f\left(\tau_{2}\right)=\frac{\tau_{2}^{2}+3 \tau_{2}+1}{\tau_{2}^{2}}
$$

and

$$
\tau_{2}>g\left(\tau_{1}\right)=\frac{\left(2 \tau_{1}+1\right)^{2}}{\tau_{1}^{3}} .
$$

If $d(G, H)=0$, the inequality to be proven is trivial. Otherwise, let us normalize $d(G, H)$ to be one, and assume $G$ lies to the left of $H$. Let $G_{0}=G$ and $H_{0}=H$. Let $G_{1}$ be the 1-gap of $K_{n}$ adjacent to $H_{0}$ on its left, and let $H_{1}$ be the 2-gap of $K_{m}$ adjacent to $G_{0}$ on its right. Let $G_{2}$ be the adjacent 1-gap of $K_{m}$ rightward from $H_{1}$, and likewise define $H_{2}, G_{3}, H_{3}, \ldots$ (see Figure 9). For $i \geq 0$ let

$$
x_{i}= \begin{cases}l\left(H_{i}\right)-l\left(G_{i+1}\right), & i \text { even } \\ r\left(G_{i+1}\right)-r\left(H_{i}\right), & i \text { odd }\end{cases}
$$

and

$$
y_{i}= \begin{cases}r\left(H_{i+1}\right)-r\left(G_{i}\right), & i \text { even }, \\ l\left(G_{i}\right)-l\left(H_{i+1}\right), & i \text { odd } .\end{cases}
$$

Let $R_{i}=d\left(G_{i}, H_{i}\right)$; then $R_{0}=1$ and $R_{i+1}=\max \left(R_{i}-x_{i}-y_{i}, 0\right)$ for $i \geq 0$. Let $R_{\infty}$ be the limit as $i$ goes to infinity of $R_{i}$. Then $d\left(K_{m}, K_{n}\right)=R_{\infty}$, so we wish to show that there is a positive lower bound on $R_{\infty}$ which depends only on $\tau_{1}, \tau_{2}$, and $t$.

In the same way as we obtained (3.2) it follows that for all $i$,

$$
x_{i+1} \leq \frac{1+t}{\tau_{1}-t} y_{i}
$$

and

$$
y_{i+1} \leq \frac{1+t}{\tau_{2}-t} x_{i}
$$

Furthermore, by Lemma 4 we have that

$$
y_{i}+x_{i+1}+y_{i+2}+\cdots \leq \sigma_{2} y_{i}
$$


and

Thus, for each $i$,

$$
x_{i}+y_{i+1}+x_{i+2}+\cdots \leq \sigma_{1} x_{i}
$$

$$
R_{\infty} \geq R_{i}-x_{i}-y_{i}-x_{i+1}-y_{i+1}-\cdots \geq R_{i}-\sigma_{1} x_{i}-\sigma_{2} y_{i} .
$$

We will show that for some $i$, the right side of (3.10) is positive.

Next let us obtain upper bounds on $x_{0}$ and $y_{0}$. We know that

$$
x_{0}=l\left(H_{0}\right)-l\left(G_{1}\right) \leq\left|G_{1}\right|+d\left(G_{1}, H_{0}\right) \leq(1+t)\left|G_{1}\right|,
$$

and by hypothesis $\left|G_{1}\right| \leq\left|G_{0}\right|$, so

$$
x_{0}=l\left(H_{0}\right)-r\left(G_{0}\right)-\left(l\left(G_{1}\right)-r\left(G_{0}\right)\right)=1-d\left(G_{0}, G_{1}\right) \leq 1-\tau_{1}\left|G_{1}\right| .
$$

Eliminating $\left|G_{1}\right|$ from these inequalities yields

$$
x_{0} \leq \frac{1+t}{\tau_{1}+1+t} .
$$

Similarly,

$$
y_{0} \leq \frac{1+t}{\tau_{2}+1+t} .
$$

We can obtain similar bounds on $x_{i}$ and $y_{i}$ for $i \geq 1$, but the bounds are complicated by the fact that we do not know in general that $\left|G_{i+1}\right| \leq\left|G_{i}\right|$ (or $\left.\left|H_{i+1}\right| \leq\left|H_{i}\right|\right)$. The analogues of (3.11) and (3.12) are thus

$$
x_{i} \leq(1+t)\left|G_{i+1}\right|
$$

and

$$
x_{i} \leq R_{i}-\tau_{1} \min \left(\left|G_{i}\right|,\left|G_{i+1}\right|\right) .
$$

If $\left|G_{i+1}\right| \leq\left|G_{i}\right|$, then as in (3.13) it follows that

$$
x_{i} \leq \frac{1+t}{\tau_{1}+1+t} R_{i}
$$

If $\left|G_{i+1}\right|>\left|G_{i}\right|$, then by (3.15),

$$
x_{i} \leq R_{i}-\tau_{1}\left|G_{i}\right| \leq R_{i}-\frac{\tau_{1}}{1+t} x_{i-1} .
$$

If (3.16) fails, then using (3.17) together with the negation of (3.16), one finds that $x_{i-1}$ is bounded above by the right side of (3.16). Thus regardless of the relative lengths of $G_{i}$ and $G_{i+1}$,

$$
\min \left(x_{i} x_{i-1}\right) \leq \frac{1+t}{\tau_{1}+1+t} R_{i},
$$

for $i \geq 1$. Likewise, regardless of the relative lengths of $H_{i}$ and $H_{i+1}$, we have for all $i \geq 1$ that

$$
\min \left(y_{i}, y_{i-1}\right) \leq \frac{1+t}{\tau_{2}+1+t} R_{i} .
$$

Let $a_{i}=x_{i} / R_{i}$ and $b_{i}=y_{i} / R_{i}$ provided $R_{i}>0$; then

$$
R_{i+1}=\max \left(1-a_{i}-b_{i}, 0\right) R_{i} .
$$


Thus $a_{i+1}$ and $b_{i+1}$ are defined as long as $1-a_{i}-b_{i}>0$. For $j=1,2$ let

$$
\lambda_{j}=\frac{1+t}{\tau_{j}+1+t}, \quad \mu_{j}=\frac{1+t}{\tau_{j}-t} .
$$

The conditions (3.13), (3.14), (3.18), and (3.19) can then be written

$$
\begin{gathered}
a_{0} \leq \lambda_{1}, \quad b_{0} \leq \lambda_{2}, \\
\min \left(a_{i+1}, \frac{a_{i}}{1-a_{i}-b_{i}}\right) \leq \lambda_{1},
\end{gathered}
$$

and

$$
\min \left(b_{i+1}, \frac{b_{i}}{1-a_{i}-b_{i}}\right) \leq \lambda_{2} .
$$

Also, conditions (3.8) and (3.9) become

$$
a_{i+1} \leq \mu_{1} \frac{b_{i}}{1-a_{i}-b_{i}}
$$

and

$$
b_{i+1} \leq \mu_{2} \frac{a_{i}}{1-a_{i}-b_{i}} .
$$

Finally, our objective is to show that for some $i$,

$$
1-\sigma_{1} a_{i}-\sigma_{2} b_{i}>0,
$$

which implies that the right side of (3.10) is positive.

We observe that $a_{i+1}$ and $b_{i+1}$ are defined at least as long as $a_{i} \leq \lambda_{1}$ and $b_{i} \leq \lambda_{2}$, because then

$$
\begin{aligned}
1-a_{i}-b_{i} & \geq 1-\lambda_{1}-\lambda_{2}=\frac{\tau_{1} \tau_{2}-(1+t)^{2}}{\left(\tau_{1}+t+1\right)\left(\tau_{2}+t+1\right)} \\
& >\frac{\left(\tau_{1}-t\right)\left(\tau_{2}-t\right)-(1+t)^{2}}{\left(\tau_{1}+t+1\right)\left(\tau_{2}+t+1\right)}>0
\end{aligned}
$$

(since $\left.t<\left(\tau_{1} \tau_{2}-1\right) /\left(\tau_{1}+\tau_{2}+2\right)\right)$. Also, as long as $a_{i} \leq \lambda_{1}$, by (3.22) we have

$$
b_{i+1} \leq \frac{\mu_{2} \lambda_{1}}{1-\lambda_{1}-b_{i}} \text {. }
$$

Let

$$
h(b)=\frac{\mu_{2} \lambda_{1}}{1-\lambda_{1}-b} .
$$

The equation $h(b)=b$ has two solutions,

$$
b_{ \pm}=\frac{1-\lambda_{1} \pm \sqrt{\left(1-\lambda_{1}\right)^{2}-4 \mu_{2} \lambda_{1}}}{2}
$$

and if the roots are real, then $h(b)<b$ for $b_{-}<b<b_{+}$(this can be verified by checking the value $\left.b=\left(1-\lambda_{1}\right) / 2\right)$. We claim that for $t$ sufficiently small, $b_{ \pm}$are real, with

$$
b_{+}>\lambda_{2}
$$

and

$$
1-\sigma_{1} \lambda_{1}-\sigma_{2} b_{-}>0
$$


Let us delay the verification of this claim until the end of the proof. Choose $b_{*}>b_{-}$with $1-\sigma_{1} \lambda_{1}-\sigma_{2} b_{*}>0$. Now $b_{0} \leq \lambda_{2}<b_{+}$, and as long as $a_{i} \leq \lambda_{1}$ continues to hold, $b_{i+1} \leq h\left(b_{i}\right)<b_{i}$ for $b_{i} \in\left(b_{-}, b_{+}\right)$. Then eventually $b_{i} \leq b_{*}$, and furthermore since $b-h(b)$ must have a positive minimum value on $\left[b_{*}, \lambda_{2}\right]$ (if $b_{*}>\lambda_{2}$ then $b_{0}<b_{*}$ already) there is a maximum number $N$ (depending only on $\tau_{1}, \tau_{2}$, and $t$ ) of iterations it can take before $b_{i} \leq b_{*}$. We therefore have shown that if $a_{i} \leq \lambda_{1}$ for $i \leq N$, then $b_{i} \leq b_{*}$ for some $i \leq N$, and hence

$$
1-\sigma_{1} a_{i}-\sigma_{2} b_{i} \geq 1-\sigma_{1} \lambda_{1}-\sigma_{2} b_{*}>0 .
$$

If on the other hand $a_{i+1}>\lambda_{1}$ for some $i \leq N$, then let $i$ be the smallest index for which this occurs. We claim that then (3.23) holds for $i$. By the results of the previous paragraph, $b_{i}<b_{i-1}<\cdots<b_{0} \leq \lambda_{2}$. Also, by (3.21), $a_{i} \leq \lambda_{1}\left(1-a_{i}-b_{i}\right)$, or in other words

$$
a_{i} \leq \frac{\lambda_{1}}{1+\lambda_{1}}\left(1-b_{i}\right)
$$

Then

$$
1-\sigma_{1} a_{i}-\sigma_{2} b_{i} \geq 1-\frac{\sigma_{1} \lambda_{1}}{1+\lambda_{1}}-\left(\sigma_{2}-\frac{\sigma_{1} \lambda_{1}}{1+\lambda_{1}}\right) b_{i}
$$

Now when $t=0$,

$$
\begin{aligned}
\sigma_{2}-\frac{\sigma_{1} \lambda_{1}}{1+\lambda_{1}} & =\frac{\left(\tau_{1}+1\right) \tau_{2}}{\tau_{1} \tau_{2}-1}-\frac{\tau_{1}\left(\tau_{2}+1\right)}{\left(\tau_{1}+2\right)\left(\tau_{1} \tau_{2}-1\right)} \\
& =\frac{\tau_{1}\left(\tau_{1} \tau_{2}-1\right)+2 \tau_{1} \tau_{2}+2 \tau_{2}}{\left(\tau_{1}+2\right)\left(\tau_{1} \tau_{2}-1\right)}>0,
\end{aligned}
$$

and thus for $t$ sufficiently small it remains positive. Then since $b_{i} \leq \lambda_{2}$,

$$
\begin{aligned}
1-\sigma_{1} a_{i}-\sigma_{2} b_{i} & \geq 1-\frac{\sigma_{1} \lambda_{1}}{1+\lambda_{1}}-\left(\sigma_{2}-\frac{\sigma_{1} \lambda_{1}}{1+\lambda_{1}}\right) \lambda_{2} \\
& =1-\sigma_{2} \lambda_{2}-\left(1-\lambda_{2}\right) \frac{\sigma_{1} \lambda_{1}}{1+\lambda_{1}} .
\end{aligned}
$$

When $t=0$, by (3.6)

$$
\begin{aligned}
1-\sigma_{2} \lambda_{2} & =1-\frac{\left(\tau_{1}+1\right) \tau_{2}}{\left(\tau_{2}+1\right)\left(\tau_{1} \tau_{2}-1\right)}=\frac{\tau_{1} \tau_{2}^{2}-2 \tau_{2}-1}{\left(\tau_{2}+1\right)\left(\tau_{1} \tau_{2}-1\right)} \\
& >\frac{\tau_{2}^{2}+3 \tau_{2}+1-2 \tau_{2}-1}{\left(\tau_{2}+1\right)\left(\tau_{1} \tau_{2}-1\right)}=\frac{\tau_{2}}{\tau_{1} \tau_{2}-1},
\end{aligned}
$$

while

$$
\left(1-\lambda_{2}\right) \frac{\sigma_{1} \lambda_{1}}{1+\lambda_{1}}=\frac{\tau_{2}}{\tau_{2}+1} \cdot \frac{\tau_{1}\left(\tau_{2}+1\right)}{\left(\tau_{1}+2\right)\left(\tau_{1} \tau_{2}-1\right)}=\frac{\tau_{1}}{\tau_{1}+2} \cdot \frac{\tau_{2}}{\tau_{1} \tau_{2}-1},
$$

so the right side of (3.27) is positive for $t=0$. It therefore remains positive for $t$ sufficiently small.

To summarize, we have shown that if $t$ is sufficiently small, then for some $i \leq N$, either (3.26) or (3.27) holds. The right side of each of these equations is positive and depends only on $\tau_{1}, \tau_{2}$, and $t$. Furthermore, $a_{j} \leq \lambda_{1}$ and $b_{j} \leq \lambda_{2}$ 
for $j \leq i$, so by (3.20), $R_{j+1} \geq\left(1-\lambda_{1}-\lambda_{2}\right) R_{j}$, and hence $R_{i} \geq\left(1-\lambda_{1}-\lambda_{2}\right)^{i}$. Then by (3.10),

$$
R_{\infty} \geq R_{i}\left(1-\sigma_{1} a_{i}-\sigma_{2} b_{i}\right) \geq\left(1-\lambda_{1}-\lambda_{2}\right)^{N}\left(1-\sigma_{1} a_{i}-\sigma_{2} b_{i}\right),
$$

where $1-\sigma_{1} a_{i}-\sigma_{2} b_{i}$ is in turn bounded below by the lesser of the right sides of (3.26) and (3.27). We have therefore shown for $t$ sufficiently small how to obtain a positive lower bound on $R_{\infty}$ which depends only on $\tau_{1}, \tau_{2}$, and $t$; we let $\psi_{t}\left(\tau_{1}, \tau_{2}\right)$ be this lower bound.

It remains for us to verify (3.24) and (3.25). We again show they are true for $t=0$, whence they hold for $t$ sufficiently small by continuity. When $t=0$,

$$
\begin{aligned}
b_{ \pm} & =\frac{\tau_{1} /\left(\tau_{1}+1\right) \pm \sqrt{\tau_{1}^{2} /\left(\tau_{1}+1\right)^{2}-4 /\left(\left(\tau_{1}+1\right) \tau_{2}\right)}}{2} \\
& =\frac{\tau_{1} \tau_{2} \pm \sqrt{\tau_{1}^{2} \tau_{2}^{2}-4\left(\tau_{1}+1\right) \tau_{2}}}{2\left(\tau_{1}+1\right) \tau_{2}}
\end{aligned}
$$

Now by (3.6),

$$
\begin{aligned}
\tau_{1}^{2} \tau_{2}^{2}-4\left(\tau_{1}+1\right) \tau_{2} & =\tau_{1}\left(\tau_{1} \tau_{2}^{2}-4 \tau_{2}\right)-4 \tau_{2} \\
& >\tau_{1}\left(\tau_{2}^{2}-\tau_{2}+1\right)-4 \tau_{2} \\
& >\frac{\left(\tau_{2}^{2}+3 \tau_{2}+1\right)\left(\tau_{2}^{2}-\tau_{2}+1\right)-4 \tau_{2}^{3}}{\tau_{2}^{2}} \\
& =\frac{\left(\tau_{2}^{2}-\tau_{2}-1\right)^{2}}{\tau_{2}^{2}}
\end{aligned}
$$

Thus $b_{ \pm}$are real and distinct (and the same must then hold for $t$ sufficiently small). Next by (3.28),

$$
b_{+}=\frac{\tau_{2}+\sqrt{\tau_{2}^{2}-4 \tau_{2}\left(1 / \tau_{1}+1 / \tau_{1}^{2}\right)}}{2 \tau_{2}\left(1+1 / \tau_{1}\right)}
$$

from which we see that $b_{+}$is increasing as a function of $\tau_{1}$. Thus $b_{+}$is greater than the value it would take on if (3.6) were an equality, which owing to (3.29) means

$$
\begin{aligned}
b_{+} & >\frac{\left(\tau_{2}^{2}+3 \tau_{2}+1\right) / \tau_{2}+\left|\tau_{2}^{2}-\tau_{2}-1\right| / \tau_{2}}{2\left(2 \tau_{2}^{2}+3 \tau_{2}+1\right) / \tau_{2}} \\
& \geq \frac{\tau_{2}^{2}+3 \tau_{2}+1-\left(\tau_{2}^{2}-\tau_{2}-1\right)}{2\left(\tau_{2}+1\right)\left(2 \tau_{2}+1\right)} \\
& =\frac{1}{\tau_{2}+1}=\lambda_{2} .
\end{aligned}
$$

Hence (3.24) holds for $t=0$, and consequently for $t$ sufficiently small.

When $t=0,(3.25)$ can be written

$$
b_{-}<\frac{1-\sigma_{1} \lambda_{1}}{\sigma_{2}}=\frac{\tau_{1}^{2} \tau_{2}-2 \tau_{1}-1}{\left(\tau_{1}+1\right)^{2} \tau_{2}}
$$

The right side of (3.30) is an increasing function of $\tau_{2}$, and since

$$
b_{-}=\frac{\tau_{1}-\sqrt{\tau_{1}^{2}-4\left(\tau_{1}+1\right) / \tau_{2}}}{2\left(\tau_{1}+1\right)},
$$


$b_{-}$is a decreasing function of $\tau_{2}$. Then by (3.7),

$$
\begin{aligned}
b_{-} & <\frac{\tau_{1}-\sqrt{\tau_{1}^{2}-4 \tau_{1}^{3}\left(\tau_{1}+1\right) /\left(2 \tau_{1}+1\right)^{2}}}{2\left(\tau_{1}+1\right)} \\
& =\frac{\tau_{1}\left(\left(2 \tau_{1}+1\right)-\sqrt{\left(2 \tau_{1}+1\right)^{2}-4\left(\tau_{1}^{2}+\tau_{1}\right)}\right.}{2\left(\tau_{1}+1\right)\left(2 \tau_{1}+1\right)} \\
& =\frac{\tau_{1}^{2}}{\left(\tau_{1}+1\right)\left(2 \tau_{1}+1\right)},
\end{aligned}
$$

while

$$
\begin{aligned}
\frac{\tau_{1}^{2} \tau_{2}-2 \tau_{1}-1}{\left(\tau_{1}+1\right)^{2} \tau_{2}} & >\frac{\left(2 \tau_{1}+1\right)^{2} / \tau_{1}-\left(2 \tau_{1}+1\right)}{\left(\tau_{1}+1\right)^{2}\left(2 \tau_{1}+1\right)^{2} / \tau_{1}^{3}} \\
& =\frac{\tau_{1}^{2}\left(2 \tau_{1}+1-\tau_{1}\right)}{\left(\tau_{1}+1\right)^{2}\left(2 \tau_{1}+1\right)}=\frac{\tau_{1}^{2}}{\left(\tau_{1}+1\right)\left(2 \tau_{1}+1\right)} .
\end{aligned}
$$

Thus (3.25) holds for $t=0$, and for $t$ sufficiently small. The proof of Lemma 5 is now complete.

\section{INTERSECTING THREE OR MORE CANTOR SETS}

In proving Theorem 1, we chose a subset $S$ of $S_{1} \cap S_{2}$ in order to guarantee positive thickness. In this section we demonstrate that positive thickness sets are in some sense generic in $S_{1} \cap S_{2}$. We also explain how Theorem 1 is useful in finding conditions under which three or more Cantor sets must have a nonempty intersection.

The set $S$ we constructed in $\S 3$ need not be dense in $S_{1} \cap S_{2}$ nor even in the nonisolated points of $S_{1} \cap S_{2}$. However, there are subsets of $S_{1} \cap S_{2}$ with thickness at least $\varphi\left(\tau_{1}, \tau_{2}\right)$ near any accumulation point. To see this, let $\left\{q_{n}\right\}$ be a sequence of distinct points in $S_{1} \cap S_{2}$ which converge to a point $q$. It is not hard to show that within any neighborhood $N$ of $q$ there are compact subsets $T_{1} \subset S_{1}$ and $T_{2} \subset S_{2}$, each of which contains all but finitely many $q_{n}$, with $\tau\left(T_{1}\right) \geq \tau\left(S_{1}\right)$ and $\tau\left(T_{2}\right) \geq \tau\left(S_{2}\right)$. Notice that any two compact sets which intersect in three or more points must be interleaved. Thus $T_{1}$ and $T_{2}$ are interleaved, and by Theorem 1 their intersection contains a set with thickness at least $\varphi\left(\tau_{1}, \tau_{2}\right)$. We conclude that arbitrarily near any nonisolated point of $S_{1} \cap S_{2}$ there are subsets of $S_{1} \cap S_{2}$ which have thickness at least $\varphi\left(\tau_{1}, \tau_{2}\right)$.

In addition to showing that there are many subsets of $S_{1} \cap S_{2}$ with positive thickness, it is possible to obtain a lower bound on the diameter of the positive thickness subset $S$ of $S_{1} \cap S_{2}$. If the two sets $S_{1}$ and $S_{2}$ are interleaved in such a way that neither is contained in the convex hull of the other, then by the discussion following the statement of Lemma 5, the diameter of $S$ is at least $\psi\left(\tau_{1}, \tau_{2}\right)$ times the length of overlap between the convex hulls of $S_{1}$ and $S_{2}$. Since the thickness of $S$ is at least $\varphi\left(\tau_{1}, \tau_{2}\right)$, we immediately have the following result.

Corollary 6. Let $S_{1}$ and $S_{2}$ be two interleaved compact sets whose thicknesses $\left(\tau_{1}, \tau_{2}\right)$ lie in region $C$ and for which the intersection $Q$ of their convex hulls contains neither $S_{1}$ nor $S_{2}$. If $S_{3}$ is a compact set with largest bounded gap $G$ such that 
(i) the hull of $S_{3}$ contains $Q$,

(ii) $|G|<\psi\left(\tau_{1}, \tau_{2}\right)|Q|$,

(iii) $\tau\left(S_{3}\right) \varphi\left(\tau_{1}, \tau_{2}\right) \geq 1$,

then $S_{1} \cap S_{2} \cap S_{3}$ is nonempty.

We note that if instead of condition (iii) we required the pair $\tau\left(S_{3}\right)$ and $\varphi\left(\tau_{1}, \tau_{2}\right)$ to lie in $C$, then $S_{1} \cap S_{2} \cap S_{3}$ would contain a set of thickness at least $\varphi\left(\tau\left(S_{3}\right), \varphi\left(\tau_{1}, \tau_{2}\right)\right)$. Thus one can inductively find thickness conditions guaranteeing the nonempty intersection of any finite (or even countably infinite) number of compact sets, although the analogue of the interleaving condition gets more complicated.

If $\left(\tau_{1}, \tau_{2}\right)$ is sufficiently far from the boundary of region $C$, then as discussed in the remark preceding the proof of Lemma 5 it is not hard to obtain explicit lower bounds on $\varphi\left(\tau_{1}, \tau_{2}\right)$ and $\psi\left(\tau_{1}, \tau_{2}\right)$. In particular, for $\tau_{1}$ and $\tau_{2}$ large we found that $\varphi\left(\tau_{1}, \tau_{2}\right)$ is at least of order $\sqrt{\min \left(\tau_{1}, \tau_{2}\right)}$, and $\psi\left(\tau_{1}, \tau_{2}\right)$ is approximately one.

We thank the referee for a thorough reading of our paper and many helpful comments.

\section{REFERENCES}

1. K. J. Falconer, The geometry of fractal sets, Cambridge Univ. Press, 1985.

2. R. Kraft, Intersections of thick Cantor sets, Mem. Amer. Math. Soc., vol 97, no. 468, 1992.

3. J. M. Marstrand, Some fundamental geometrical properties of plane sets of fractional dimensions, Proc. London Math. Soc. (3) 4 (1954), 257-302.

4. P. Mattila, Hausdorff dimension and capacities of intersections of sets in n-space, Acta Math. 152 (1984), 77-105.

5. S. Newhouse, Nondensity of axiom $A(a)$ on $S^{2}$, Proc. Sympos. Pure Math., vol. 14, Amer. Math. Soc., Providence, R.I., 1970, pp. 191-202.

6. __ Diffeomorphisms with infinitely many sinks, Topology 13 (1974), 9-18.

7. __ The abundance of wild hyperbolic sets and non-smooth stable sets for diffeomorphisms, Inst. Hautes Etudes Sci. Publ. Math. 50 (1979), 101-151.

8. __ Lectures on dynamical systems, Progress in Math. 8, Birkhäuser, Boston, Mass., 1980, pp. 1-114.

9. C. Robinson, Bifurcation to infinitely many sinks, Comm. Math. Phys. 90 (1983), 433-459.

10. R. F. Williams, How big is the intersection of two thick Cantor sets?, Continuum Theory and Dynamical Systems (M. Brown, ed.), Proceedings of the 1989 Joint Summer Research Conference on Continua and Dynamics, Amer. Math. Soc., Providence, R.I., 1991.

Code R44, Naval Surface Warfare Center, Silver Spring, Maryland 20903-5000

Current address: Institute for Physical Science and Technology, University of Maryland, College Park, Maryland 20742

E-mail address, B. R. Hunt: hunt@ ipst.umd.edu 22030

Department of Mathematical Sciences, George Mason University, Fairfax, Virginia

E-mail address, I. Kan: ikan@gmu.edu

Institute for Physical Science and Technology, Mathematics, University of Maryland, College Park, Maryland 20742

E-mail address, J. A. Yorke: yorke@ipst.umd.edu 